\title{
SUCCESSFUL THERAPY OF CLOSTRIDIUM DIFFICILE INFECTION WITH FECAL MICROBIOTA TRANSPLANTATION
}

\begin{abstract}
'Department of Medicine, Thuringia Clinic Saalfeld, Teaching Hospital of the University Jena Saalfeld/Saale, Germany; ${ }^{2}$ Department of Microbiology, Faculty of Biochemistry, Biophysics and Biotechnology, Jagiellonian University, Cracow, Poland; ${ }^{3}$ Department of Medicine I, University Erlangen-Nuremberg, Erlangen, Germany
\end{abstract}

\begin{abstract}
Clostridium difficile infection (CDI) is the most common cause of infectious diarrhea and represents an important burden for healthcare worldwide. Symptoms of severe CDI include watery, foul-smelling diarrhea, peripheral leucocytosis, increased C-reactive protein (CRP), acute renal failure, hypotension and pseudomembranous colitis. Recent studies indicate that the main cause of CDI is dysbiosis, an imbalance in the normal gut microbiota. The restoration of a healthy gut microbiota composition via fecal microbiota transplantation (FMT) recently became more popular. The aim of the present study was to assess the effect of FMT on the healing of CDI and to analyze the changes in the level of pro-inflammatory markers (C-reactive protein, fecal calprotectin) and pro-inflammatory cytokines. Eighteen patients with CDI were included in our study (6 males and 12 females) with recurrent and/or severe CDI. The FMT was performed in 17 patients using colonoscopy, including 16 patients receiving a one-time FMT and 1 patient who needed 2 additional FMTs. One patient was treated with a single round of FMT using push-and-pull enteroscopy. In all CDI patients, before and 3 weeks after FMT, the following parameters were analyzed: C-reactive protein, fecal calprotectin, and plasma interleukin (IL)-6, IL-8 and IL-12, and tumor necrosis factor-alpha (TNF- $\alpha$ ). In addition, the plasma level of LL-37, a cathelicidine peptide was assessed by fluorescence-activated cell sorting (FACS) before and 3 months after FMT. Finally, in 7 patients a microbiome analysis was performed by sequencing of 16SrRNA in stool probes obtained before and 3 weeks after FMT. The healing rate of CDI was $94 \%$. In all successfully treated patients no recurrent CDI was observed during follow-up (16 months). The serum level of pro-inflammatory cytokines (TNF- $\alpha$, IL$1 \beta$, IL-6, IL-8 and IL-12) significantly decreased after FMT. Similarly, CRP and fecal calprotectin normalized after FMT. 3 months after FMT a significant increase of LL-37 in the plasma of successfully treated patients was monitored. The sequencing analysis demonstrated an elevated abundance of beneficial bacterial species such as Lactobacillaceae, Ruminococcaceae, Desulfovibrionaceae, Sutterellaceae and Porphyromonodacea after FMT. No serious side effects were observed. We concluded that FMT represented a very effective and safe treatment of recurrent and/or severe CDI and led to favorable shifts in the composition of gut microbiome.
\end{abstract}

Key words: Clostridum difficile infection, fecal microbiota therapy, gut microbiota, C-reactive protein, fecal calprotectin, LL-37, interleukin-1 $\beta$, interleukin-6, interleukin-8, interleukin-12, tumor necrosis factor-alpha, fluorescence-activated cell sorting

\section{INTRODUCTION}

Gut microbiota plays a crucial role in the preservation of intestinal homeostasis, the development of the immune system and the host nutrition metabolism, and represents an important defense line against gastrointestinal infections (1).

In recent years, a huge number of studies demonstrated that gut microbiota might be modified by different factors including genetics, geography, diet, hygiene, aging, stress exposure and drugs that may impair the diversity and composition of the gut flora and promote the development of a dysbiosis (2).

As a consequence, dysbiosis can lead to intestinal and extraintestinal diseases, among these the Clostridium difficile infection (CDI). CDI represents one of the most prevalent hospital-acquired bacterial infections in the developed world. Recent data indicate an increase in both the incidence and the severity of CDI (2).

The clinical presentation of CDI ranges from asymptomatic carriage to fulminant colitis. Most frequently the patients infected with toxin-producing $C$. difficile develop colitis that is accompanied by non-bloody diarrhea, fever, abdominal pain or discomfort. Severe cases are characterized by increased white cell count over $15,000 / \mu \mathrm{L}$, hypoalbuminemia and elevated serum creatinine level. Very severe CDI can lead to dangerous complications such as toxic megacolon or spontaneous colon perforation. Epidemiological data indicate that over the last years the mortality and morbidity caused by CDI are rising leading to prolonged hospitalization and higher therapy costs $(4,5)$. 
From the clinical point of view, the treatment of CDI is hampered by an increasing prevalence of antibiotic resistance and frequent recurrences (6). The therapy is adjusted to the severity of the disease. In mild or moderate cases of CDI, the antibiotic therapy is applied for $10-14$ days with either metronidazole at a dose $500 \mathrm{mg}$ on prescription or vancomycin at a dose $125 \mathrm{mg}$ four times a day. For a severe or complicated form of CDI, fidaxomycin (dose 200mg two times a day) should be used (7).

From another episode of the infection suffer $10-20 \%$ of CDI patients. The likelihood of developing further CDI episodes increases to $40-65 \%$ if the patient already had one recurrence of the disease $(6,7)$.

Due to intense research on the field of the gut microbiota, we now know that CDI is a common consequence of intestinal dysbiosis. Repeated antiobiotic therapy has a negative impact on the gut microbiom diversity and thus increases the risk for further overgrowth of $C$. difficile and the probability of CDI reccurences $(6,8)$. The only way to break this circulus vitiosus seems to be the restoration of gut microbiota with fecal microbiota transplantation (FMT). FMT is an emerging therapy for CDI that offers the potential for a rapid and lasting elimination of the infection through restoration of healthy microbiota. FMT is a process of stool transfer from one or multiple healthy donors to the gut of the sick person (9).

Although this method has been already used in ancient medicine, Eiseman and collegues were the first who treated patients with $C$. difficile via fecal enema and published the results in 1958 (10). The first very promising, placebo-controlled trial by Van Nood et al., confirmed the high effectivity of this method in the treatment of CDI (11). Further meta-analyses demonstrated a cure rate of over $90 \%$ in CDI patients treated with FMT (12). The effectiveness of FMT in patients with CDI provides important proof of concept that dysbiotic gut microbiota can be restored by transplantation of microbiota from a healthy donor.

The aim of the present study was: 1) to analyze the impact of fecal microbiota transplantation (FMT) on the healing and the recurrence rate of $C$. difficile infections; 2) to assess the effect of FMT on the imbalance of pro-inflammatory cytokines and other pro-inflammatory markers C-reactive protein (CRP) and fecal calprotectin $(\mathrm{Fc})$, and 3 ) to study the shift in gut microbiome diversity and composition after FMT.

\section{PATIENTS AND METHODS}

\section{Patients}

This study included 18 patients with CDI. Fifteen of them had recurrent CDI and 3 severe and/or complicated CDI. All patients who provided informal consent were recruited for this study undertaken in the Thuringia Clinic Saalfeld between March 2014 and October 2016. The male:female ratio was $6: 12$. The median age of patients was 66.9 years. During a stay in hospital $78 \%$ of the patients aquired CDI. In $22 \%$ of the patients CDI occured in an ambulant setting. The severity of CDI was assessed on the basis of the Hines VA CDI severity score index (13).

\section{Experimental procedures}

The preferred method of FMT application was colonoscopy. The screening of the donor and the preparation of the FMT was based on the modified Amsterdam protocol (14). Only in one patient the upper gastrointestinal (GI) route via double-balloon enteroscopy was chosen since colonoscopy was inapplicable due to sigma stenosis. The main advantages of the colonoscopic route are: 1) colonoscopy is a more pleasant method for the patient than upper gastrointestinal enteroscopy, 2) colonoscopy offers the ability to evaluate the lower gastrointestinal tract and to exclude other pathologies, and 3) the colonoscopic route allows a higher bacterial load for FMT which may increase the effectiveness of this method. The median follow up was 16 months (3 weeks - 31 months). The total number of FMTs applied was 20 with one patient receiving 3 FMTs over a period of 8 months.

For each patient included in our study the following laboratory parameters were assessed before and after FMT: the complete blood count, the level of CRP, and the presence $C$. difficile toxin in stool samples. In 8 patients, the analysis of fecal calprotectin was performed. Furthermore, in 8 patients the levels of pro-inflammatory cytokines such as TNF- $\alpha$, IL-1 $\beta$, IL6 , IL- 8 and IL-12 was measured by FACS analysis. Brieflly, serum level of TNF- $\alpha$, IL-1 $\beta$, IL-6, IL- 8 and IL-12 was assessed by multiplex measurements using the Human Inflammatory Cytokine CBA Kit (BD Biosciences, Cat. No: 551811). Data weas acquired on BD ${ }^{\text {TM}} F A C S$ Calibur flow cytometer, and analysed using FCAP Array Software. Moreover, the level of LL-37 peptide was estimated by ELISA (Hycult Biotech).

In 7 patients the analysis of diversity and composition of gut microbiota was assessed by using sequencing of 16sRNA. Shortly, genomic DNA was extracted according to Qiamp Fast DNA Stool extraction kit (Qiagen) and the V3-V4 region of bacterial 16srRNA genes was amplified with the NEBNext Q5 Hot Start Hifi PCR Master Mix (NEB) using a dual-index strategy (15). The amplified fragments were purified with AMPure XP Beads (Beckmann Coulter Genomics), combined and analyzed by ' $2 \times 300$ bp paired-end' sequencing using a Illumina MiSeq device. Quality control and bioinformatics analysis was done using the Uparse Pipeline (16) and Megan 6 (17). The database of the 'Ribosomal database project' (RDP, release 15) was used for classification.

\section{Statistical analysis}

All statistical analyses were performed using SPSS version 22.0 (SPSS, Inc, Chicago IL, USA). Non-normally distributed data are presented as medians and categorical data are presented as $\mathrm{n}(\%)$. Outcomes before and after treatment were compared using the Wilcoxon rank sum test. $\mathrm{P}<0.05$ was considered to be statistically significant.

\section{RESULTS}

After application of one FMT a $88.8 \%$ cure rate of CDI was achieved, since 16 of 18 patients responded to FMT. For one patient, two additional FMT were needed to achieve a definitive long-lasting cure. Only one patient with severe CDI did not respond to FMT. Therefore, the final outcome of the study was $94.4 \%$. Regarding the indication for FMT after recruitment for the study, 15 patients had recurring CDI and 3 patients had a first episode of severe, therapy-refractory C. difficile enteritis (Fig. 1).

No serious short- or long-term adverse events were observed. Within the first $8-12$ hours after FMT 10 of 17 patients complained about abdominal discomfort that completely disappeared $24 \mathrm{~h}$ after the procedure. No fever or allergic reactions were observed.

During the mean follow-up period of 16 months (March 2014 to October 2016), no recurrence of CDI was observed in all successfully treated patients. One patient with peripheral arterial disease died 3 weeks after FMT due to infected ischemic 
necrosis of the lower extremity with consecutive sepsis. Three other patients died during the follow-up due to non-FMTreleated diseases, but also showed no recurrent CDI symptoms until their death; two patients died 6 months after FMT due to myocardial infarction or apoplex, and one patient died due to severe chronic obstructive pulmonary disease (COPD) stage IV and pneumonia 3 months after FMT.

The FMT therapy led to a significant reduction of CRP in all treated patients (Fig. 2). In parallel to the reduction of CRP, the normalization of the clinical score was observed (Fig. 3). In 8 CDI patients the fecal calprotectin level was assessed. This sensitive marker of colonic inflammation significantly decreased after FMT (Fig. 4).

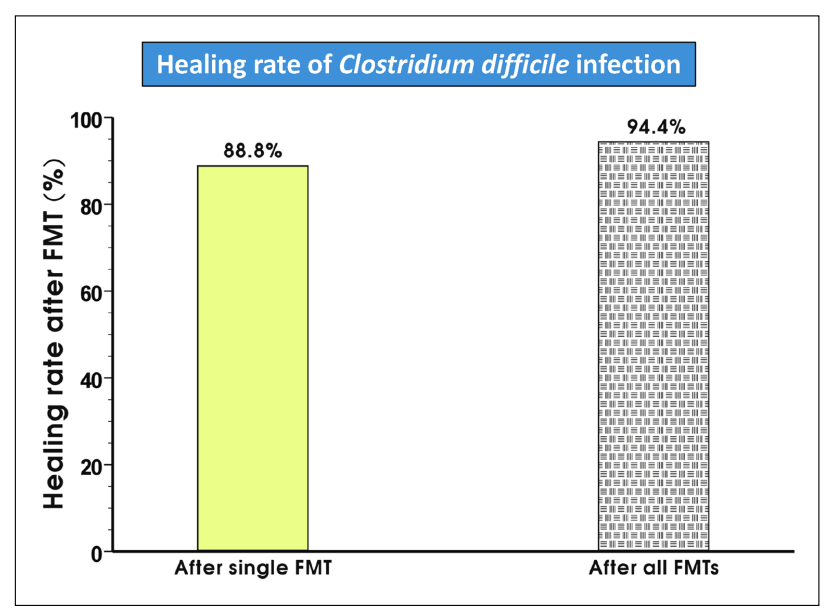

Fig. 1. Healing rate of Clostridium difficile infection (CDI) after one FMT and after all FMTs.
The analysis of pro-inflammatory cytokines by FACS analysis demonstrated a significant decline of the level of TNF$\alpha$, IL-1 $\beta$, IL-6, IL-8 and IL-12, already 3 weeks after the FMT procedure (Fig. 5).

Cathelicidins are important anti-microbial peptides involved in the protection of the intestinal barrier. In animal model of sepsis, cathelicidin prevented intestinal barrier dysfunction (18). LL-37 is the only cathelicidin-derived anti-microbial peptide found in humans. Our analysis showed no significant change in the LL-37 level 3 weeks after FMT; however, the amount of the peptide in the patients' plasma significantly increased within 3 months after the FMT procedure. These results point towards the restoration of the intestinal barrier after the healing of CDIinduced colitis (Fig. 6).

Finally, the analysis of the diversity of gut microbiota using the Shannon index demonstrated a significant increase in gut microbiome diversity after FMT (Fig. 7).

In 7 representative patients with recurrent $\mathrm{CDI}$, the sequencing analysis of gut microbiota using the 16sRNA sequencing technique was performed. This examination showed a significant increase of beneficial bacterial species such as Lactobacillaceae, Ruminococcaceae, Desulfovibrionaceae, Sutturellaceae and Porhyromonadeacae, and a decrease in the bacterial community of Enterobacteriaceae and Veillonellaceae (Fig. 8).

\section{DISCUSSION}

Our study demonstrated the high effectivity of fecal microbiota transplantation (FMT) therapy in patients with recurrent and/or severe $C$. difficile colitis. The cure rate in our FMT-treated patients was $94 \%$. Our data are conform to the results obtained in previous studies (12).

\section{Course of the C-reactive protein concentrations in individual patients}

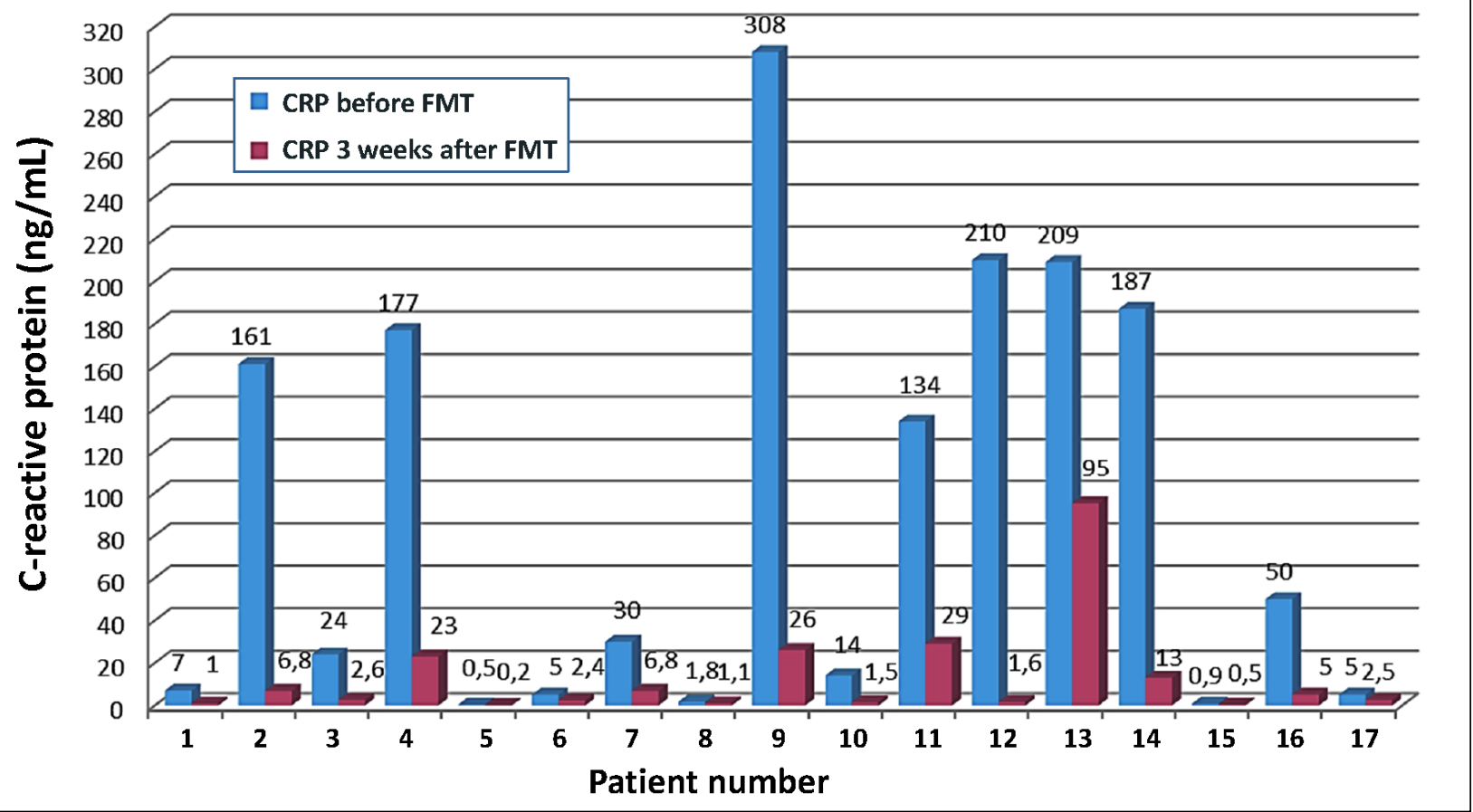

Fig. 2. Changes in C-reactive protein in patients with Clostridium difficile infection before and 3 weeks after FMT. 


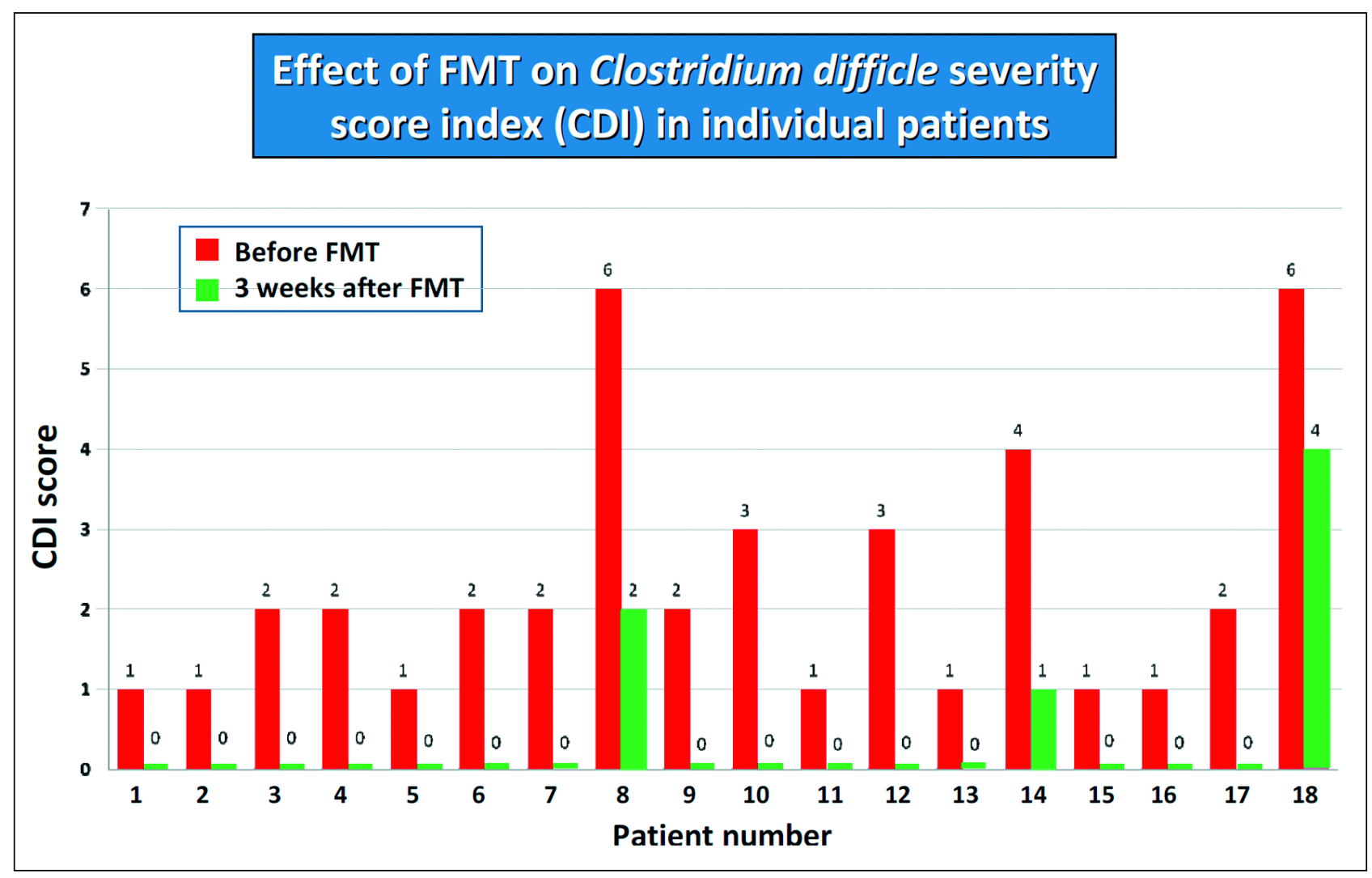

Fig. 3. Clinical score of CDI before and 3 weeks after FMT.

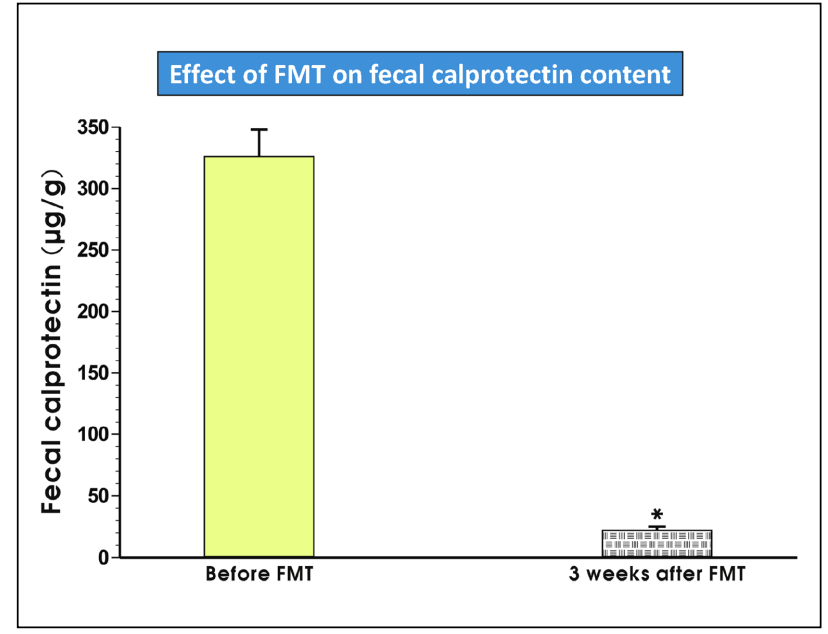

Fig. 4. Median value of fecal calprotectin before and 3 weeks after FMT.

In one patient with severe, pseudomembranous, therapyrefractory CDI the application of FMT failed. This indicates that a severe, pseudomembranous CDI may not respond to FMT. A recent study by Fischer et al. demontrated that severe CDI, accompanied by the development of pseudomembranes, is associated with increased risk for failure of FMT (19).

The therapy was well tolerated and no serious adverse events were observed. During the follow-up period of 16 months we registered 4 deaths which were not related to FMT; the study participants died of sepsis, myocardial infarction, apoplex or severe COPD with pneumonia. No allergic reaction or fever were noted after FMT. The most common adverse event after fecal microbiota therapy was abdominal discomfort, as reported also in a previous study (20).

Our study showed that during the follow-up no reccurences of CDI were observed indicating an improvement of dysbiosis through FMT that prevents a repeated overgrowth of $C$. difficile and a new outbreak of this disease.

The main route of the application in our study was colonoscopy. In only 1 patient FMT was successfully perfomed via double-balloon enteroscopy due to sigma stenosis and a risk for gut perforation during colonoscopy. FMT via the colonoscopic route seems to be highly effective since colonoscopy offers the possibility to apply more fecal material and thus a higher concentration of bacterial communities to the diseased colon. Our data hint towards that the colonoscopic route for FMT should be favored over FMT applied enteroscopically. Additionally, enteroscopy harbors the risk of severe aspiration and limits the use of the upper gastrointestinal tract as a path for the treatment of FMT $(20,21)$.

In the present study, FMT caused a rapid attenuation of colonic mucosa inflammation. Within 3 weeks after FMT, CRP and fecal calprotectin completely normalized in CDI patients. This observation indicates that FMT therapy imposes strong anti-inflammatory effects due to the transfer of a 'healthy' bacterial communities. This hypothesis is supported by the fact that the levels of the pro-inflammatory cytokines TNF- $\alpha$, IL $1 \beta$, IL6, IL-8 and IL-12 significantly dropped within 3 weeks after FMT, leading to the normalization of the CDI score. To the best of our knowledge, this is the first report describing beneficial changes of plasma pro-inflammatory cytokines in patients after successful therapy of CDI with FMT.

As previously shown, the use of FMT in CDI patients was associated with a significant increase in bacterial diversity that indicates the restoration of gut microbiota (22). Moreover, we were able to identify the bacterial species responsible for anti- 
inflammatory effects in the colonic mucosa. The sequencing technique of 16sRNA showed a significant increase in the number of anti-inflammatory bacteria such as Lactobacillaceae, Ruminococcaceae, Desulfovibrionaceae, Sutturellaceae and Porhyromonadeacae, and a decrease in aggresive bacterial communities such as Enterobacteriaceae and Veillonellaceae.

\section{Effects of FMT on the plasma cytokine levels}

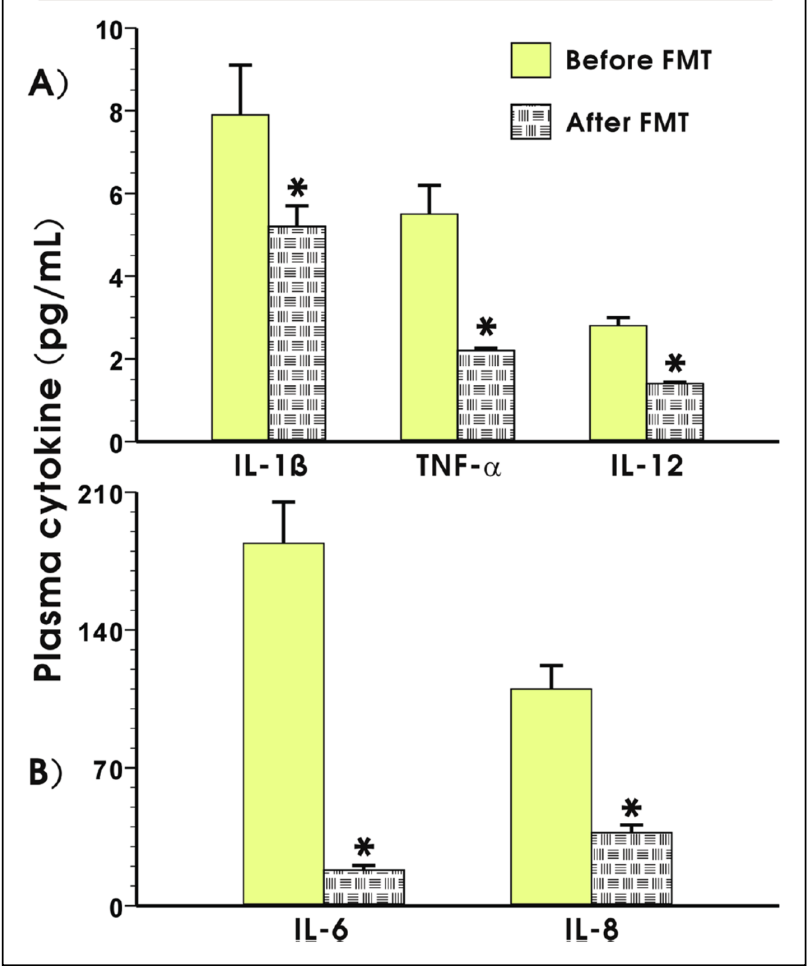

Fig. 5. Plasma level of IL-1 $\beta$, TNF- $\alpha$, IL-6, IL- 8 and IL-12 before and 3 weeks after FMT.
Lactobacillaceae represents a large family of rod-shaped or spherical gram-negative bacteria that require carbohydrates for growth and ferment them mainly to lactic acid. This group of bacteria protects the gut mucosa and ameliorates colitis by inhibition of the nuclear factor kappa-light-chain-enhancer of activated $\mathrm{B}$ cells $(\mathrm{NF}-\mathrm{\kappa B})$ and strengthening of the intestinal barrier via induction of tight junction protein expression (23). Ruminococcaceae are common gut microbes that break down complex carbohydrates. Recently, Khanna et al. demonstrated that an increase in Ruminococcaceae in association with other bacterial communities (Clostridiaceae, Rikenellaceae, Bacteroides, Rothia and/or Faecalibacterium) is observed in the gut of the patient who respond to treatment of $C$. difficile infection (24). Desulfovibrionaceae are sulfate-reducing bacteria (SRB) that produce the important mediator hydrogen sulfide; $\mathrm{H}_{2} \mathrm{~S}$ plays an important role in the human gut because it exerts vasodilatory, neuromodulatory and anti-inflammatoty effects

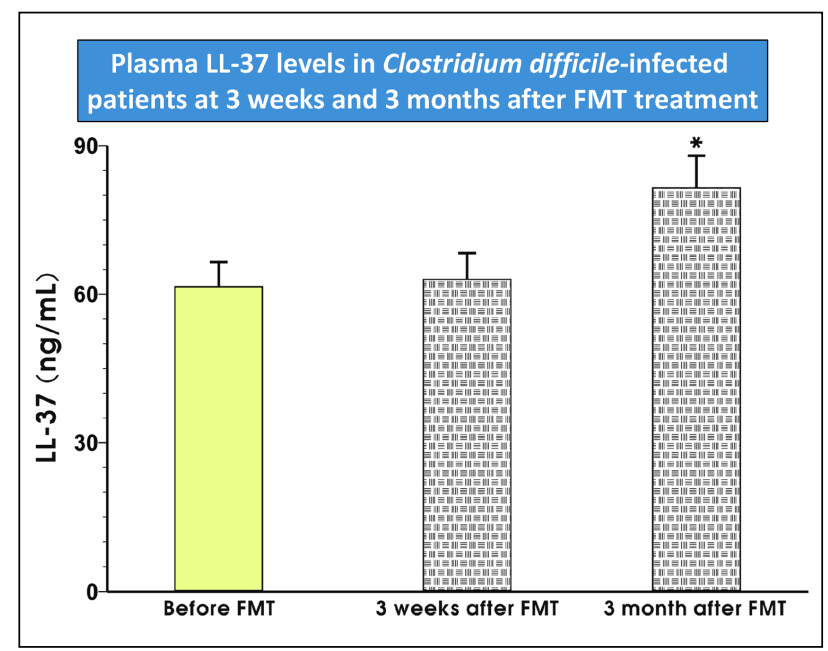

Fig. 6. Changes in plasma level of cathelicidine protein LL-37 before and 3 weeks and 3 months after FMT in patients with CDI.

\section{Assessment of $\alpha$-diversity and composition of gut microbiota in selected patients with Clostridium difficile infection}

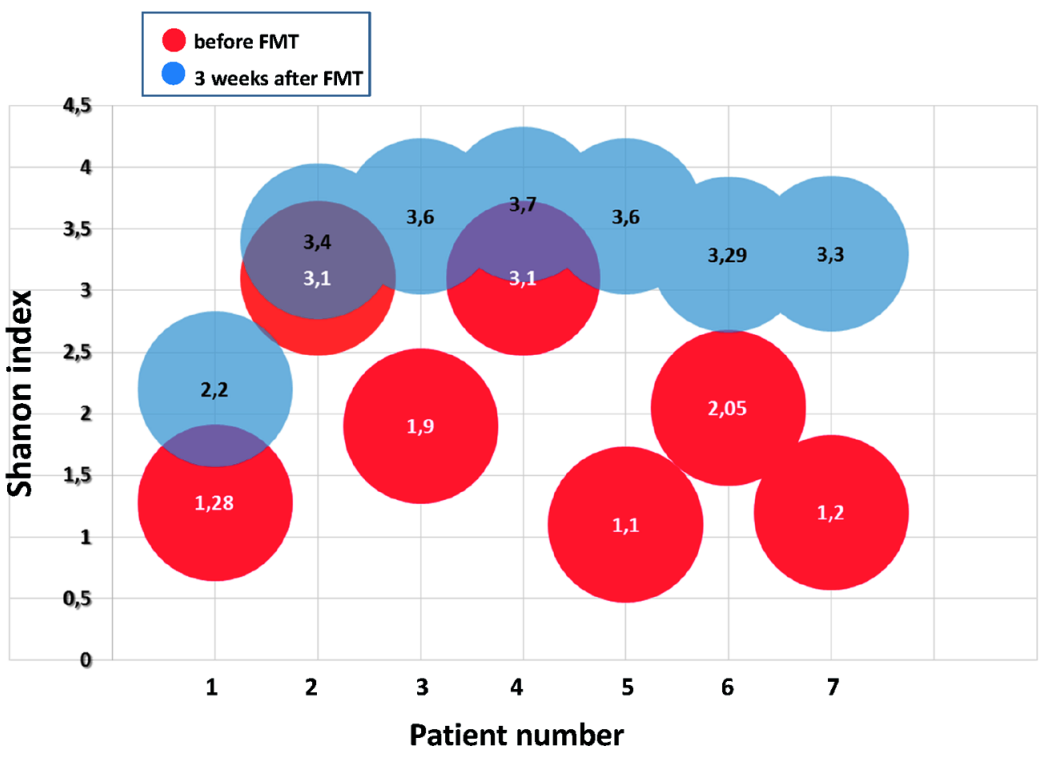

Fig. 7. Alpha-diversity of gut microbiome expressed as Shannon index before and 3 weeks after FMT in patients with CDI. 


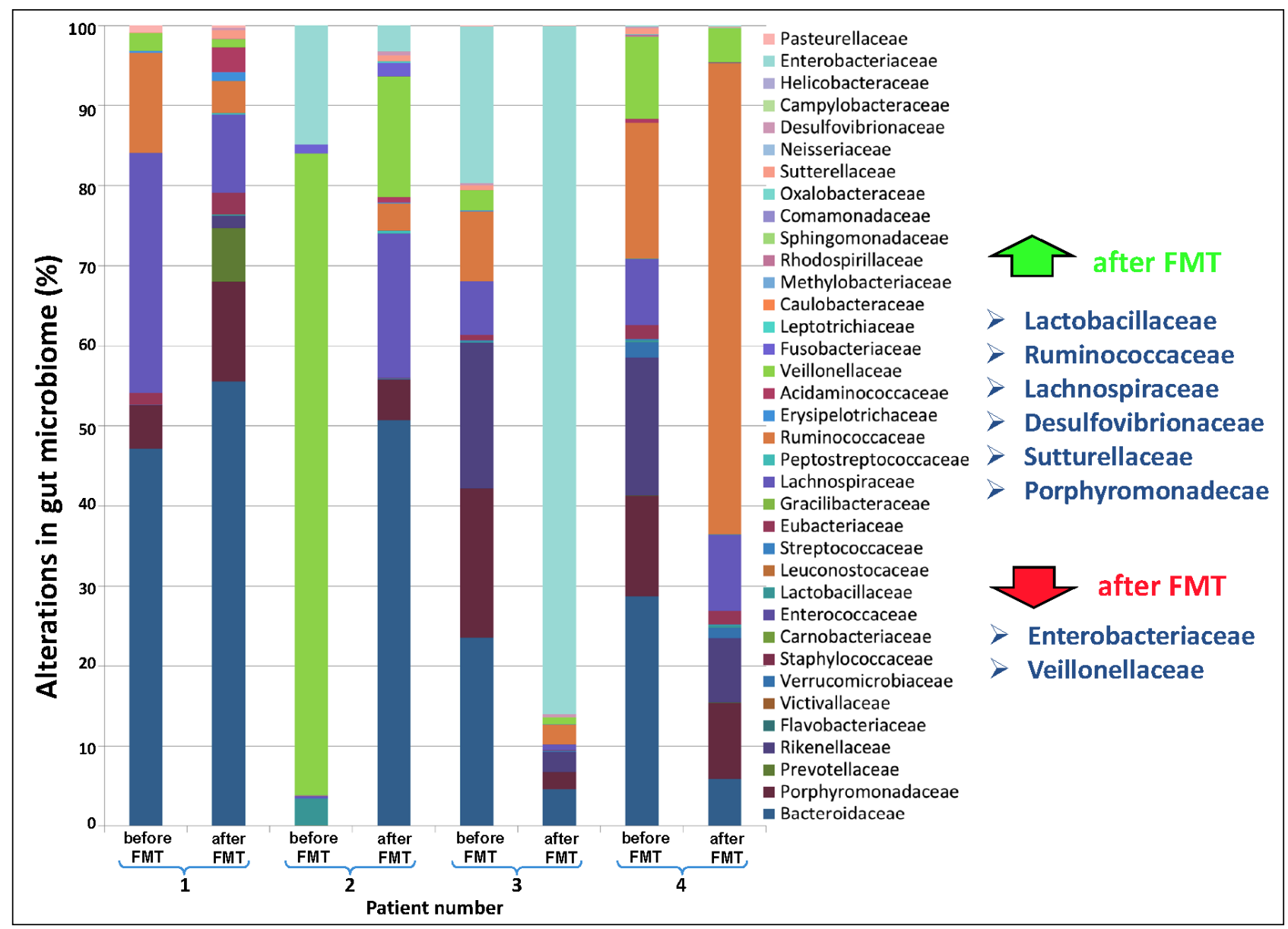

Fig. 8. Changes in gut microbiome in 4 patients with CDI before and after FMT assessed by 16sRNA sequencing technique.

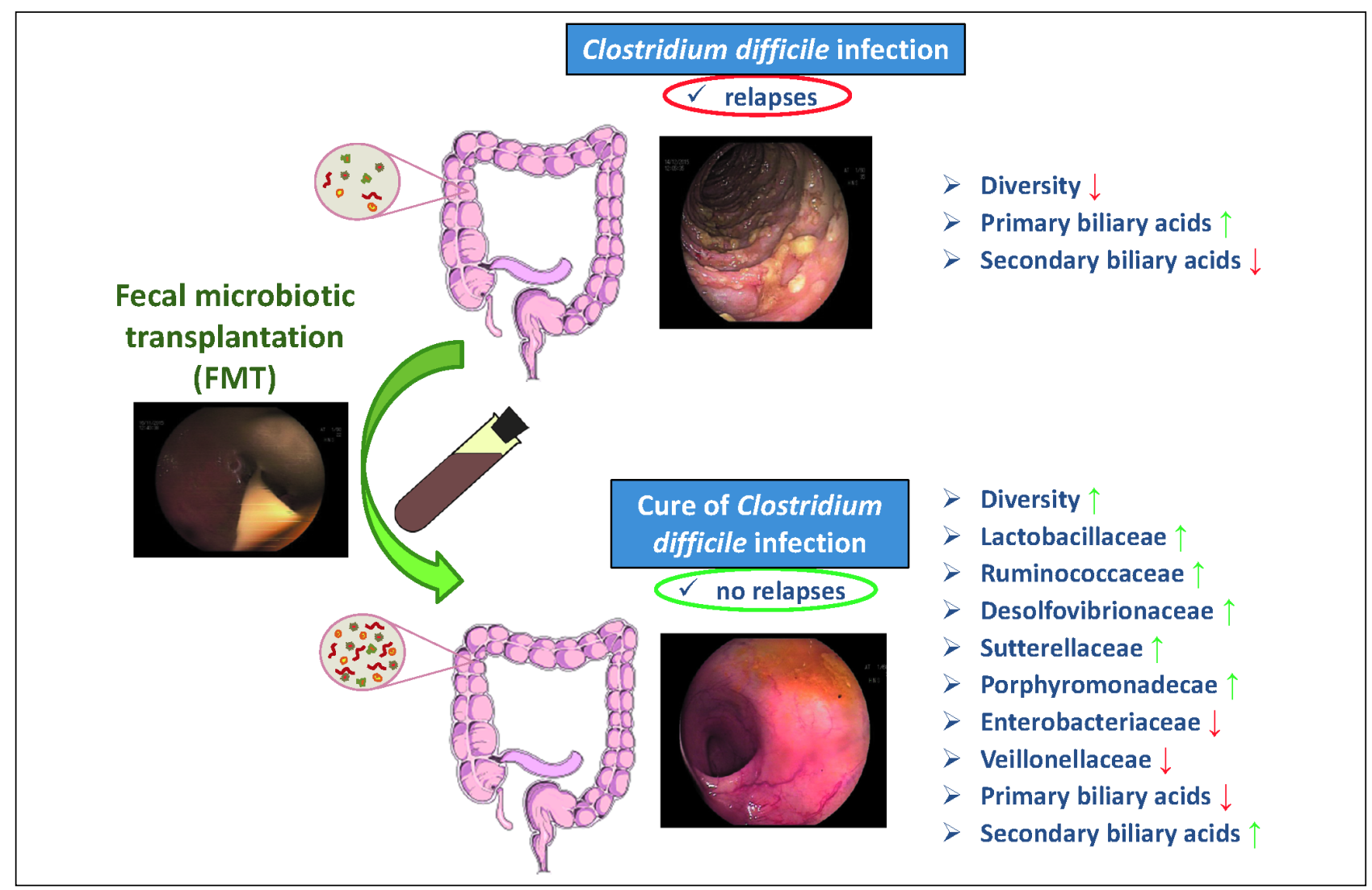

Fig. 9. Schematic presentation of the beneficial effect of FMT in patients with CDI. 
$(25,26)$. The role of Sutturellaceae is not defined yet and is under investigation. The ability of these bacteria to adhere to intestinal epithelial cells indicate that they may have an immunomodulatory role (27).

Porhyromonadeacae is a family within the order of Bacteroidales and represent an important part of the indigenous microbiota of the gastrointestinal tract and the oral cavity. Recently, it has been demonstrated that Porhyromonadeacae increase colonization resistance against $C$. difficile in a murine model of this infection (28).

Although the present study did not investigate the metabonomic changes after FMT, previous studies indicate that FMT is associated with increased production of secondary bile acids by the gut bacteria that are inhibitory to C. difficile (29).

Finally, our results demonstrated that the overall microbiota diversity as shown by Shannon index increases after FMT. These results agree with previous observations (30).

From our study, we conclude that 1) fecal microbiota transplantation (FMT) is an effective treatment method for $C$. difficile infection (CDI) since $>90 \%$ of the participating patients showed no recurrence of the disease; 2) successful FMT is accompanied by a significant reduction of CRP, proinflammatory cytokines and fecal calprotectin; and 3) FMT seems to be very effective and safe treatment of recurrent and/or severe CDI and leads to favorable shifts in the composition of gut microbiome (Fig. 9).

Conflict of interests: None declared.

\section{REFERENCES}

1. Sun J, Chang EB. Exploring gut microbes in human health and disease: pushing the envelope. Genes Dis 2014; 1: 132-139.

2. Sommer F, Backhed F. The gut-microbiota-masters of host development and physiology. Nat Rev Microbiol 2013; 11: 227-238.

3. Konturek PC, Haziri D, Brzozowski T, et al. Emerging role of fecal microbiota therapy in the treatment of gastrointestinal and extra-gastrointestinal diseases. J Physiol Pharmacol 2015; 66: 483-491.

4. Martin JS, Monaghan TM, Wilcox MH. Clostridium difficile infection: epidemiology, diagnosis and understanding transmission. Nat Rev Gastroenterol Hepatol 2016; 13: 206-216.

5. Magee G, Strauss ME, Thomas SM, Brown H, Baumer D, Broderick KC. Impact of Clostridium difficile-associated diarrhea on acute care length of stay, hospital costs, and readmission: a multicenter retrospective study of inpatients, 2009-2011. Am J Infect Control 2015; 43: 1148-1153.

6. Bouza E. Consequences of Clostridium difficile infection: understanding the healthcare burden. Clin Microbiol Infect 2012; 18 (Suppl. 6): 5-12.

7. Ofozu A. Clostridium difficile infection: a review of current and emerging therapies. Ann Gastroenterol 2016; 29: $147-154$

8. Marchesi JR, Adams DH, Fava F, et al. The gut microbiota and host health: a new clinical frontier. Gut 2016; 65: 330-339.

9. Kelly CR, Kahn S, Kashyap P, et al. Update on fecal microbiota transplantation 2015: indications, methodologies, mechanisms, and outlook. Gastroenterology 2015; 149: 223-237.

10. Eiseman B, Silen W, Bascom GS, Kauvar AJ. Fecal enema as an adjunct in the treatment of pseudomembranous enterocolitis. Surgery 1958; 44: 854-859.
11. Van Nood E, Vrieze A, Nieuwdorp M, et al. Duodenal infusion of donor feces for recurrent Clostridium difficile. $N$ Engl J Med 2013; 368: 407-415.

12. Li YT, Cai HF, Wang ZH, Xu J, Fang JY. Systematic review with meta-analysis: long-term outcomes of faecal microbiota transplantation for Clostridium difficile infection. Alim Pharmacol Ther 2016; 43: 445-457.

13. Fujitani S, George WL, Murthy AR. Comparision of clinical severity score indices for Clostridium difficile infection. Infect Control Hosp Epidemiol 2011; 32: 220-228.

14. Keller JJ, Kuijper EJ. Treatment of recurrent and severe Clostridium difficile infection. Annu Rev Med 2015; 66: 373-386.

15. Fadrosh DW, Ma B, Gajer $\mathrm{P}$, et al. An improved dualindexing approach for multiplexed 16S rRNA gene sequencing on the Illumina MiSeq platform. Microbiome 2014; 2: 6. doi: 10.1186/2049-2618-2-6.

16. Edgar RC. UPARSE: highly accurate OTU sequences from microbial amplicon reads. Nat Methods 2013; 10: 996-998.

17. Huson DH, Weber N. Microbial community analysis using MEGAN. Methods Enzymol 2013; 531: 465-485.

18. Song D, Zong X, Zhang H, et al. Antimicrobial peptide cathelicidin-BF prevents intestinal barrier dysfunction in a mouse model of endotoxemia. Int Immunopharmacol 2015; 25: 141-147.

19. Fischer M, Kao D, Mehta SR, et al. Predictors of early failure after fecal microbiota transplantation for the therapy of Clostridium difficile infection: a multicenter study. Am J Gastroenterol 2016; 111: 1024-1031.

20. Wang S, Xu M, Wang W, et al. Systematic review: Adverse events of fecal microbiota transplantation. PLoS One 2016; 11: e0161174. doi: 10.1371/journal.pone.0161174

21. Link A, Lachmund T, Schulz C, Weigt J, Malfertheiner P. Endoscopic peroral jejunal fecal microbiota transplantation. Dig Liver Dis 2016; 48: 1336-1339.

22. Kelly CR, Khortus A, Staley C, et al. Effect of fecal microbiota transplantation on recurrence in multiply recurrent Clostridium difficile infection: a randomized trial. Ann Intern Med 2016; 165: 609-616.

23. Jeong JJ, Kim KA, Jang SE, Woo JY, Han MJ, Kim DH. Orally administrated Lactobacillus pentosus var. plantarum C29 ameliorates age-dependent colitis by inhibiting the nuclear factor-kappa B signaling pathway via the regulation of lipopolysaccharide production by gut microbiota. PLoS One 2015; 10: e116533. doi: 10.1371/journal.pone. 0116533

24. Khanna S, Montassier E, Schmidt B, et al. Gut microbiome predictors of treatment response and recurrence in primary Clostridium difficile infection. Alim Pharmacol Ther 2016; 44: 715-727.

25. Zhang-Sun W, Augusto LA, Zhao L, Caroff M. Desulfovibrio desulfuricans isolates from the gut of a single individual: structural and biological lipid A characterization. FEBS Lett 2015; 589: 165-171.

26. Magierowski M, Jasnos K, Kwiecien S, et al. Endogenous prostaglandins and afferent sensory nerves in gastroprotective effect of hydrogen sulfide against stressinduced gastric lesions. PLoS One 2015; 10: e0118972. doi: 10.1371/journal.pone.0118972

27. Hiippala K, Kainulainen V, Kalliomaki M, Arkilla P. Satokari R. Mucosal prevalence and interactions with the epithelium indicate commensalism of Sutterella spp. Front Microbiol 2016;7: 1706.

28. Schubert AM, Sinani H, Schloss PD. Antibiotic-induced alterations of the murine gut microbiota and subsequent effects on colonization resistance against Clostridium difficile. MBio 2015; 6: e00974. doi: 10.1128/mBio.00974-15 
29. Weingarden AR, Chen C, Bobr A, et al. Microbiota transplantation restores normal fecal bile acid composition in recurrent Clostridium difficile infection. Am J Physiol Gastrointest Liver Physiol 2014; 306: G310-G319.

30. Girotra M, Garg S, Anand R, Song Y, Dutta SK. Fecal microbiota transplantation for recurrent Clostridium difficile infection in elderly: long term outcomes and microbiota changes. Dig Dis Sci 2016; 61: 3007-3015.

Received: August 30, 2016

Accepted: December 19, 2016

Author's address: Prof. Peter C. Konturek, Department of Medicine, Thuringia Clinic Saalfeld, Teaching Hospital of the University Jena, 68 Rainweg Street, 07318 Saalfeld/Saale, Germany.

E-mail: pkonturek@web.de 